\title{
Development of a mobile optical system to detect road-freezing conditions
}

\section{Nuerasimuguli ALIMASI ${ }^{1}$, Shuhei TAKAHASHI ${ }^{1}$ and Hiroyuki ENOMOTO}

1 Kitami Institute of Technology, Kitami 090-8507, Japan

2 National Institute of Polar Research, Tachikawa, Tokyo 190-8518, Japan

(Received March 20, 2011; Revised manuscript accepted June 13, 2012)

\begin{abstract}
Road surface freezing is a serious problem for road traffic in the winter in cold regions. This is especially true in mountainous regions, where the winter season is longer and roads are frequently covered with snow and/or ice. We sought to develop a detector for road-freezing conditions, consisting of optical sensors and an infrared thermometer. The measurement system has a light source with an incident angle of $45^{\circ}$ and two photodiodes set with receiving angles of $0^{\circ}$ (at the zenith) and $45^{\circ}$ to measure the diffuse reflection and the specular reflection (mirror reflection). Using observations from a portable measurement system, road conditions (dry, wet, 'sherbet,' compacted snow, glossy compacted snow, black ice) were distinguished well in relation to the specular reflectance $R_{\mathrm{S}}$ and the diffusive reflection $R_{\mathrm{D}}$. A mobile measurement system was installed at the rear of a car, and continuous observations were made along Route 39 around Sekihoku Pass, Hokkaido. The observed reflection data showed reasonable results for wet and sherbet surfaces along the lower parts of the course and for compacted snow and glossy compacted snow around Sekihoku Pass. The ratio of specular reflectance to diffuse reflectance, $\gamma_{\mathrm{SD}}\left(=R_{\mathrm{S}} / R_{\mathrm{D}}\right)$, enhanced the indication of the existence of black ice on the road surface, because $R_{\mathrm{S}}$ was large and $R_{\mathrm{D}}$ was small.
\end{abstract}

Key words: road surface freezing, optical sensor, specular reflection, diffuse reflection

\section{Introduction}

A slippery road surface is a serious problem for winter traffic in cold regions. A snow-covered road becomes slippery and dangerous for driving. Roads in mountainous areas are especially dangerous as the winter period is longer and the varying road surfaces can bewilder a driver, leading to confusion about the conditions found on a partly frozen road over a bridge, a road under a snow-shed, and a road in the shade.

Real-time monitoring and data services for drivers are desirable for safe winter driving. If an optical sensor on a vehicle can detect the level of slipperiness and indicate dangerous levels while driving, the probability of an accident can be decreased significantly. In this study, we sought to develop a road-freezing detector system.

Road conditions vary quickly in time and space, and frequent observations are required to provide accurate information for a driver. If we could measure road surface conditions continuously, we could detect dangerous but invisible conditions like "black ice."

There have been many studies of winter road surfaces and friction measurements. Wallman and
Åström (2001) summarized road friction measurements and traffic safety in many countries. They listed many devices used in European countries for friction measurement, which typically are based on a reference tire and wheel for measuring the friction force. Al-Qadi et al. (2002) also reviewed devices for friction measurement, as follows. Deceleration devices measure the deceleration of a vehicle under full braking. Locked-wheel devices, like the VDOT Locked Wheel Trailer system, measure the skid number. Side force devices test a wheel at some angle to the direction of motion. Fixed slip devices operate between 10 and $20 \%$ slip, and are used as runway friction testers (RFTs); these include the Airport Surface Friction Tester (ASFT), the Saab Friction Tester (SFT), and the Griptester. There are other slip devices, such as the ROAR friction trailer and SALTAR friction-measuring device.

Nonstad (2002) examined several friction measurement techniques used for winter road maintenance in Norway. The devices included decelerometers, like the C- $\mu$-trip and ELTRIP, a small portable continuous friction tester; the ASFT T2Go; and the RoAR Mark III trailer device. Nonstad (2002) also noted several new systems now available. As compact sys- 
tems, decelerometers are portable and inexpensive sensors that measure deceleration by braking and estimate road friction. However, the operator and type of car can influence the measurements. Because they need braking or speed changes for measurements, they are difficult to operate in crowded traffic conditions for safety reasons.

Some advanced systems can also be equipped with a camera that takes pictures when the friction falls below a specified level. The pictures can show observed friction changes and indicate relevant points. However, point information is not sufficient for a driver, and locality and area variability are also useful information.

BMT Fleet Technology Limited (2008) reported studies on runway friction characteristic measurements and aircraft braking. The listed friction devices were locked-wheel testers for spot measurements and side-force testers, fixed-slip testers, and variable-slip testers on towed trailers for continuous records. Hamilton (2008) summarized practical field friction testing devices, and grouped them into five categories: 1) deceleration devices used with regular anti-lock braking system (ABS) cars, 2) locked-wheel testers (100\% slip), 3) side-force devices or constant slip angle devices, 4) fixed-slip devices (normally between 10 and $20 \%$ slip), and 5) variable slip devices (0 to $100 \%$ slip). He reviewed activities in many countries. In Sweden, a friction evaluation car (with ABS and deceleration measurement equipment) can be regularly calibrated against a BV11, a Saab Friction tester, or a BV14. In the U.S., locked-wheel testers are used for general friction testing in more than 40 states (Al-Qadi et al., 2002). In Japan, the standard device is a bus full-locked wheel tester (LWFT), which is used primarily for research purposes because of its high cost (Tokunaga et al., 2008).

Feng and $\mathrm{Fu}$ (2008) reported evaluations of two new Vaisala sensors for road-surface condition monitoring. They compared road-surface condition data reported by the Vaisala sensors with those from traditional in situ pavement sensors and visual observations. Additionally, grip levels, estimated by Vaisala sensors, were compared with real surface friction measurements taken using a continuous friction meter. They found that 1) the Vaisala sensors were reliable and accurate in determining road surface contaminants, 2) there were systematic differences in temperature measurements between the Vaisala sensor and the traditional in situ friction sensors, and 3) grip levels reported by the Vaisala sensor did not correlate well with the observed friction measurements.

Haavasoja and Pilli-Sihvola (2010) reported friction measurement results from the Vaisala Remote Road Surface State Sensor DSC111 installed on a research car. The DSC111 friction correlated well with the accelerometer readings obtained by occasional lock brak- ing. They concluded that an accelerometer system was a convenient, economic, reliable, and readily installed method of obtaining friction measurements.

In Japan, Matsushima et al. (2007) summarized winter road conditions and traffic problems. Funahashi et al. (2007) and Takahashi et al. (2010) explored friction measurement devices for winter road maintenance. Hanatsuka et al. (2009) tested a new "sensing tire" device, in which an accelerometer was installed inside a tire and acceleration data were sent to a car remotely.

The friction measurement devices described above are important and useful for winter road maintenance, but are not appropriate for installation in private cars. We intended to make a lighter and cheaper sensor to detect slippery frozen surfaces using an indirect measurement system. There are several indirect devices for measuring friction forces, like the Vaisala Sensor DSC111.

Some sensors have been developed to measure road conditions using devices what people cannot see. Such sensors can provide useful information. From experience, we know that slight changes in the reflected light at the road surface can inform us about the presence of black ice. If we can obtain such information objectively, it can be provided to the driver. In this study, we attempted to use the multi-directional difference in reflected light as optical information. Detection of a reflection change is possible for drivers, using light from the headlights of oncoming cars or from street lamps. These observations enables us forecasting of road condition changes at a distance.

Important points to be considered are which type of road should be monitored and how the monitor can be configured on a car. This study aimed, as a first priority, at detecting dangerous and largely invisible road conditions; in particular, we sought especially to detect black ice.

Combinations of available compact sensors may be one solution for providing a hybrid driver support system. In this study, we aimed to contribute to such a system. Although road maintenance vehicles are typically used for this purpose, portable road-freezing detecting sensors for any vehicle will provide an advantage for road safety control. Our purpose was to try to make a readily installable sensor for private cars. We used an optical method as an indirect measuring system for convenience, and have previously reported some preliminary experiments (Alimasi and Takahashi, 2009, Takahashi and Alimasi, 2009).

Generally, a glossy surface is slippery. A black ice surface can be dangerous because a driver sometimes does not recognize its existence. We intended to detect such a glossy surface optically to reduce the danger of car accidents. This report provides the first steps in the development of a road-freezing detector system. 


\section{Measurement principles}

\subsection{Optical properties of frozen roads}

A driver often tries to recognize slippery road conditions by the glossy nature of the road surface using visual observation, which is typically easier at night even in the distance because glossy surfaces mirror the light from headlights of oncoming cars, but can sometimes be difficult to detect during the daytime. We sought to measure the gloss level automatically and provide this information for an alarm system to detect slippery road surfaces.

When a light beam impinges on a surface, the reflected light can be classified into either diffusive or specular reflection. Diffusive reflection has homogeneous intensity in all directions, as seen on a new snow surface (Fig. 1 (a)). Specular reflection, also called mirror reflection, follows reflection laws like light on a mirror surface, so that light is reflected only at the same angle as the incident angle (Fig. 1 (b)). On an actual road surface, both components of reflection will occur (Fig. 1 (c)), and we intended to observe them.

\subsection{Notation}

The Japanese Industrial Standard (JIS) (Japanese Standard Association, 1997) gives the following definitions for specular glossiness measurement methods of macroscopically smooth surfaces for products in the mining and manufacturing industries.

Specular reflection: The reflection of light following the laws of reflection, such as reflection on a specular surface.

Diff use reflection: The diffusing reflection of light except specular reflection.

Specular reflectance: The ratio of the radiant flux of reflection (or reflection of luminous flux) to the radiant flux of incidence (or luminous flux of incidence).
Specular gloss: The visual sensory attribute determined primarily by the intensity of specular reflection light.

Specular glossiness: The measured degree of specular gloss expressed in numerical values.

The standard for specular reflection at a specified incident angle $\theta$ is the value on a glass surface of refractive index $n=1.567$ constant over the range of visible wavelengths, which is expressed as $100 \%$ of each angle. As shown in Table 1 from JIS (1997), the specular reflectance $R_{0}$ increases with the incident angle $\theta_{1} . \quad R_{0}$ at $85^{\circ}$ is more than ten times larger than that at $20^{\circ}$.

The specular glossiness depends on the refractive index $n$, as shown in Table 2, and is $100 \%$ on a glass surface of $n=1.567$. By extrapolating these values, the glossiness is $46 \%$ for an ice surface $(n=1.333$ for a wavelength of $589.3 \mathrm{~nm}$ ) and $41 \%$ for a water surface $(n=1.309)$.

\subsection{Measurement tests with a gloss meter}

We tested a small commercial gloss meter (Handy Gloss Checker IG-331, Horiba Ltd.), the sensor part of which measured $\mathrm{W} 88 \times \mathrm{H} 30 \times \mathrm{D} 45 \mathrm{~mm}$. It was easy to measure glossiness at incident angles of $20^{\circ}$ and $60^{\circ}$, as long as the sensor was in contact with the surface. However, when we tried to measure the glossiness of a frozen road surface, the gloss meter hardly reacted at $20^{\circ}$ to any frozen surface tested. At $60^{\circ}$, the gloss meter showed low values on a smooth ice surface, but not on a glossy compacted snow surface. These tests indicated that we needed a more sensitive measurement system for a practical road surface device.

Because specular reflectance increases with incident angle (Table 1), a large incident/receiving angle is better for more sensitive measurements, but a large angle is not good for practical measurements on a road surface because the mobile measurement device needs to be some distance from the measurement surface, typically about $20 \mathrm{~cm}$, and it is difficult to

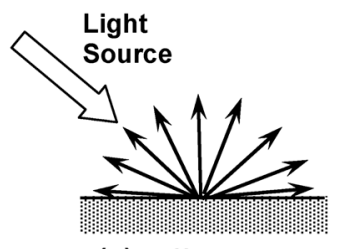

(a) Diffuse

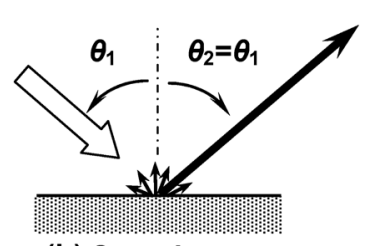

(b) Specular

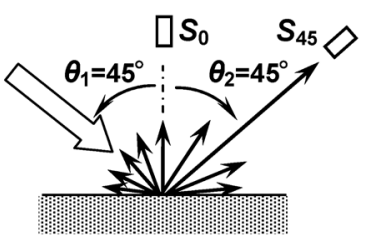

(c) Mixed reflection

Fig. 1. Concept of specular reflection and diffuse reflection: (a) diffuse reflection, (b) specular reflection $\left(\theta_{1}\right.$ is the incident angle and $\theta_{2}$ is the reflection angle), (c) mixed reflection $\left(\theta_{1}=\theta_{2}=45^{\circ}\right.$ and sensors were set at $0^{\circ}$ for diffuse reflection, and at $45^{\circ}$ for specular reflection).

Table 1. Relationship between the specular reflectance $R_{0}$ of the standard glass surface (refractive index $=1.567$ ) and incident angle $\theta_{1}$ (from JIS, 1997).

\begin{tabular}{lccccc}
\hline Incident angle: $\theta_{1}$ & $20^{\circ}$ & $45^{\circ}$ & $60^{\circ}$ & $75^{\circ}$ & $85^{\circ}$ \\
\hline Specular reflectance: $R_{0}$ & 0.0491 & 0.0597 & 0.1001 & 0.2646 & 0.6191 \\
\hline
\end{tabular}


Table 2. Relationship between specular glossiness and refractive index $n$ with incident angle $\theta_{1}$. The glossiness is defined as $100 \%$ for a glass surface with refractive index $n=1.567$. The values are from JIS Z8741 (1997).

\begin{tabular}{cccccc}
\hline Ref. index & \multicolumn{5}{c}{ Incident angle (= receiving angle) } \\
$n$ & $\theta_{1}=20^{\circ}$ & $45^{\circ}$ & $60^{\circ}$ & $75^{\circ}$ & $85^{\circ}$ \\
\hline 1.400 & 57.0 & 61.3 & 71.9 & 87.4 & 96.6 \\
1.500 & 82.0 & 84.1 & 89.1 & 95.6 & 99.0 \\
1.567 & 100.0 & 100.0 & 100.0 & 100.0 & 100.0 \\
1.600 & 109.1 & 107.9 & 105.2 & 101.9 & 100.4 \\
1.700 & 137.6 & 132.2 & 120.3 & 106.8 & 101.2 \\
1.800 & 167.0 & 156.6 & 134.6 & 110.7 & 101.7 \\
\hline
\end{tabular}

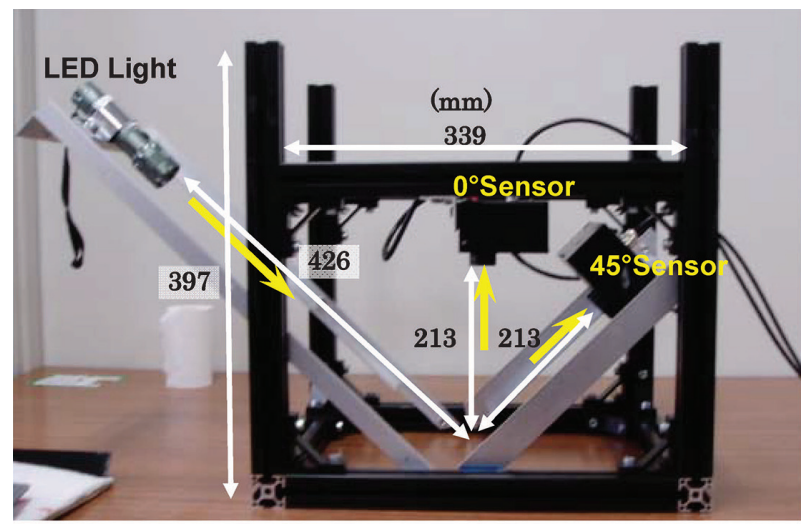

(a)

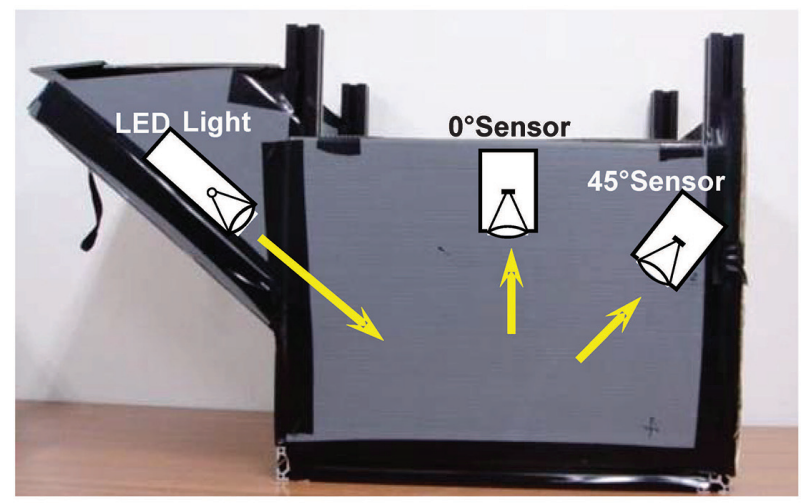

(b)

Fig. 2. Portable reflection measurement system. (a) LED light and photodiode sensor. (b) System covered with boards.

focus on the surface in a large incident/receiving angle when the distance is changing. A small incident/receiving angle is better for the focusing, but the finite size of the light source and optical sensors make some limitation for the small angle. As a result, we adopted $45^{\circ}$ for the incident/receiving angle and tried to design a measurement system for mobile observations given these issues.

\section{Portable reflection measurement system}

\subsection{Measurement system}

We designed the portable reflection measurement

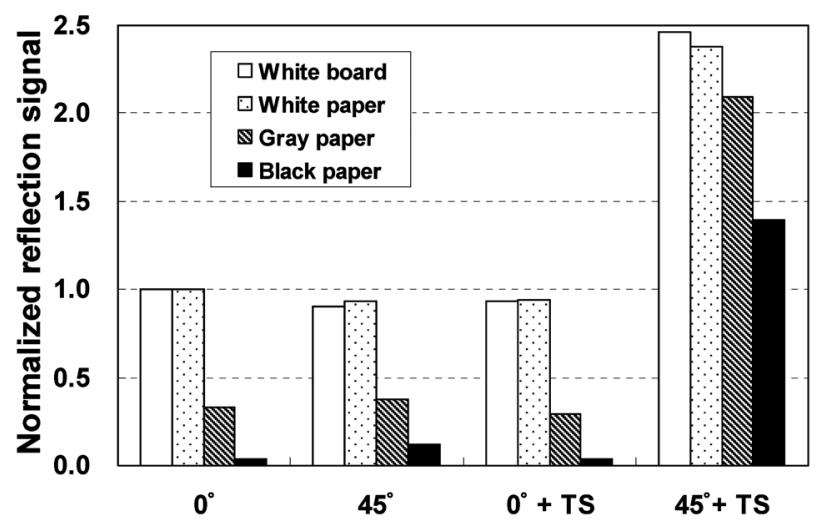

Fig. 3. Virtual experiments for a slippery road surface. " 0 " and " $45^{\circ}$ " indicate the reflection signals for the $0^{\circ}$ and $45^{\circ}$ sensors from a white standard board, white paper, gray paper, and black paper. " $0^{\circ}+$ TS "and " $45^{\circ}+$ TS" indicate experiments when a transparent sheet (TS) was overlaid on the paper to simulate a slippery surface.

system shown in Fig. 2. The light source was a single light-emitting diode (LED) light, which generated almost parallel light, $5 \mathrm{~cm}$ in diameter. A reflected light sensor was designed with a photodiode $(5 \times 5$ $\mathrm{mm}$ ) for visible wavelengths and a lens ( $28 \mathrm{~mm}$ diameter and $30 \mathrm{~mm}$ focal distance). The lens was focused on the photodiode, making a sensing area of about 30 $\mathrm{mm}$ in diameter. The signal of the photodiode was amplified using a pre-amplifier with amplification factors of 1,10 , and 100 .

The light was set at an incident angle of $45^{\circ}$ to the measurement surface at the bottom of system. The photodiode sensors were set at $0^{\circ}$ (normal to the surface) to detect diffuse reflections and at a $45^{\circ}$ reflection angle to detect specular reflection (Fig. 1(c)).

\subsection{System calibration}

First, we checked the measurement system using combinations of colored paper and transparent sheets. The transparent sheet (TS) for an overhead projector was used as a model of a smooth and slippery road surface, which has a high gloss level. The results of a laboratory experiment are shown in Fig. 3. The values in Fig. 3 are normalized to the reflection signal of a white standard plate at zero, and “+ TS" indicates 
that a TS was laid over the paper.

As shown in Fig. 3, the reflection of $0^{\circ}$ depended on the color brightness; the reflection was almost 1 for white paper, 0.4 for gray paper, and almost 0 for black paper. For reflection at $45^{\circ}$, gray paper and black paper showed values somewhat larger than the $0^{\circ}$ reflection, indicating that the paper had some glossiness. Although the reflection of " $0{ }^{\circ}+$ TS" was almost the same as that of $0^{\circ}$, the reflection of " $45^{\circ}+$ TS" showed very large values, markedly higher than the values of $0^{\circ}$ for all cases.

From these results we defined the reflection variables from the signals of $V_{0}$ at $0^{\circ}$ and $V_{45}$ at $45^{\circ}$ as follows:

$$
\begin{aligned}
& S_{0} \text { : Normalized signal at } 0^{\circ}, \\
& \quad S_{0}=V_{0} / V_{0 \mathrm{~W}},
\end{aligned}
$$

where $V_{0 \mathrm{w}}$ is the reflection signal of a white standard plate at $0^{\circ}$.

$$
\begin{aligned}
& S_{45}: \text { Normalized signal at } 45^{\circ}, \\
& \quad S_{45}=\mathrm{V}_{45} / V_{0 \mathrm{w}}, \\
& R_{\mathrm{D}}: \text { diffuse reflectance, } \\
& R_{\mathrm{D}}=S_{0} \\
& R_{\mathrm{S}}: \text { Specular reflectance, } \\
& \quad R_{\mathrm{S}}=S_{45}-S_{0}=\left(\mathrm{V}_{45}-V_{0}\right) / V_{0 \mathrm{w}}
\end{aligned}
$$

$\gamma_{\mathrm{SD}}$ : Specular reflection ratio (a ratio of $R_{\mathrm{S}}$ to $R_{\mathrm{D}}$ ), $\gamma_{\mathrm{SD}}=R_{\mathrm{S}} / R_{\mathrm{D}}=\left(V_{45}-V_{0}\right) / V_{0}$

By these definitions, $R_{\mathrm{D}}$ indicates "whiteness" which is 1 on a white surface and 0 on a black surface, $R_{\mathrm{S}}$ is specular reflection component in received reflection which becomes large on glossy surface and 0 when no specular reflection, and $\gamma_{\text {SD }}$ enhances a glossy and dark surface like a black ice surface (thin transparent ice on a paved road surface).

\subsection{Road-freezing conditions}

We classified the road surface into eight different types, as follows. Photographs of some typical road surfaces are shown in Fig. 4.

1) Dry: dry surface without water, snow, or ice.

2) Wet: wet surface with water.

3) Snow: a snowy surface with soft and uncompacted snow.

4) Sherbet: a surface with a mixture of snow and water, like sherbet.

5) Freezing: a surface with ice, which appears after wet snow is frozen.

6) Compacted snow: a surface with snow compacted by cars.

7) Glossy compacted snow: a glossy surface with compacted snow.

8) Black ice: a surface with thin ice, which looks dark.

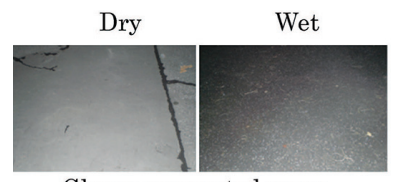

Glossy compacted snow

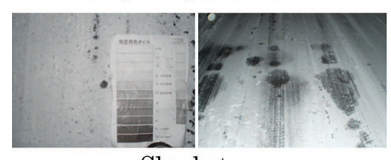

Sherbet

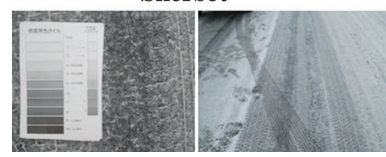

Fig. 4. Road surface conditions classified by color patterns in the Sekihoku Pass area.

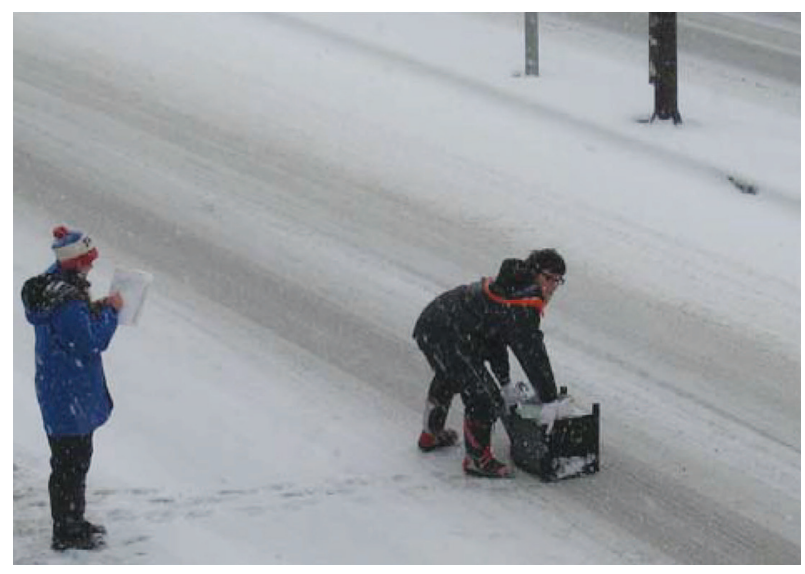

Fig. 5. Observations using the portable measurement system at Sekihoku Pass on National Route 39 on December 28, 2008.

\subsection{Observation of road surfaces}

Using the portable reflection measurement system, reflections from the road surface were measured in a mountainous area along National Route 39, in Hokkaido, around Sekihoku Pass (1050 m a.s.l) on December 6, 2008. As shown in Fig. 5, the measurement system was put on a road surface, and was in close contact with the surface to exclude outside light as much as possible. The outputs of the two photodiodes were measured using circuit testers as the LED light was turned on and off. These measurements were performed quickly in the absence of traffic.

The relationship between the specular reflectance $R_{\mathrm{S}}$ defined by Eq. (4) and the diffuse reflectance $R_{\mathrm{D}}$ defined by Eq. (3) is shown in Fig. 6. The specular reflectance $R_{\mathrm{S}}$ of black ice and glossy compacted ice surfaces ranged in relatively high values from 0.05 to 0.36 , while that of compacted snow was small, less than 0.05 . The diffuse reflectance $R_{\mathrm{D}}$ was high on the surface of compacted snow and glossy compacted snow, and low on dry, wet, sherbet, and black ice surfaces, reflecting the whiteness of the surface.

The relationship between the specular reflection ratio $\gamma_{\mathrm{SD}}$, defined by Eq. (5), and the diffuse reflectance 


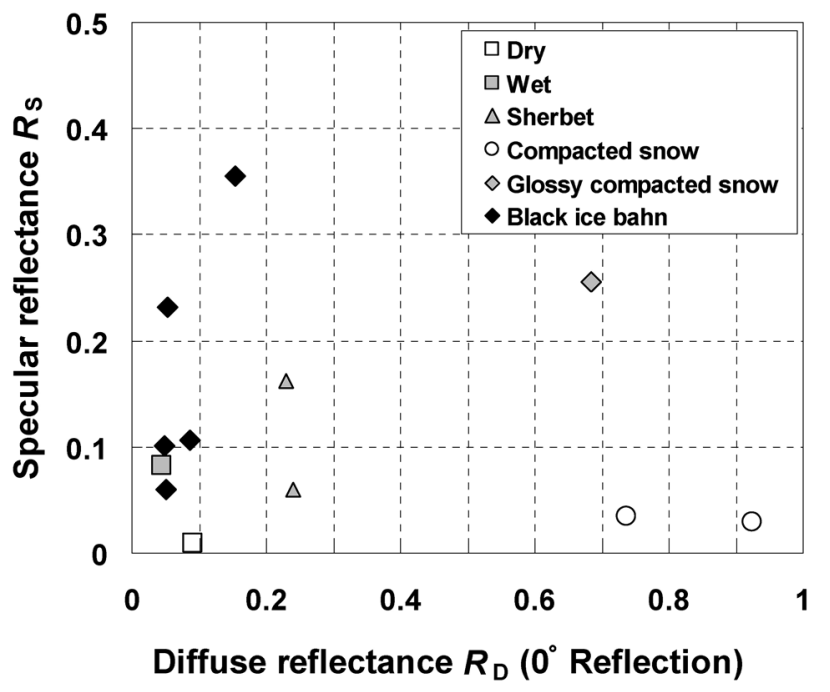

Fig. 6. Specular reflectance $R_{\mathrm{S}}\left(S_{45}-S_{0}\right)$ and diffuse reflectance $R_{\mathrm{D}}$ ( $=S_{0}: 0^{\circ}$ reflection) of a road surface around Sekihoku Pass on December 6, 2008.

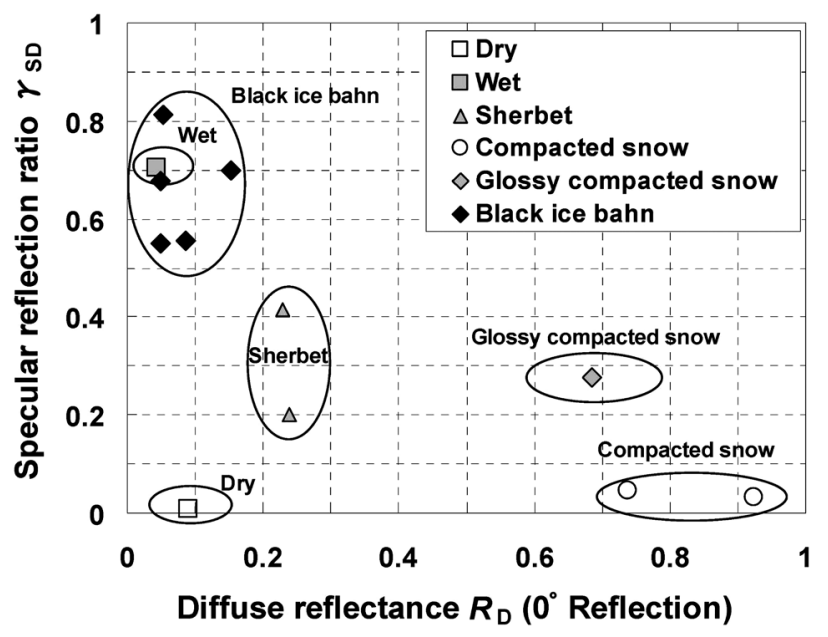

Fig. 7. Specular reflection ratio $\gamma_{\mathrm{SD}}\left(=R_{\mathrm{S}} / R_{\mathrm{D}}\right)$ and diffuse reflectance $R_{\mathrm{D}}$ on December 6, 2008 .

$R_{\mathrm{D}}$ is shown in Fig. 7. The ratio $\gamma_{\mathrm{SD}}$ tended to be high on a dark surface because of the small $R_{\mathrm{D}}$. $\gamma_{\mathrm{SD}}$ was larger on black ice than on glossy compacted snow, enhancing the indication of the existence of black ice. The ratio was higher, $0.5-0.8$, on black ice, $0.2-0.4$ on sherbet and glossy compacted snow, and lower than 0.1 on compacted snow and dry surfaces. The data for the wet surface were included in the data group with black ice, but if we know the surface temperature, we can distinguish wet from black ice.

\section{Mobile observations from a car}

\subsection{Measurement system}

For our reflection observations, the portable system described in Section 3 was improved to allow a 20 -cm space between the optical measurement system and the road surface. The same photodiodes were installed as optical sensors. The system was installed at the rear of a car (Fig. 8).

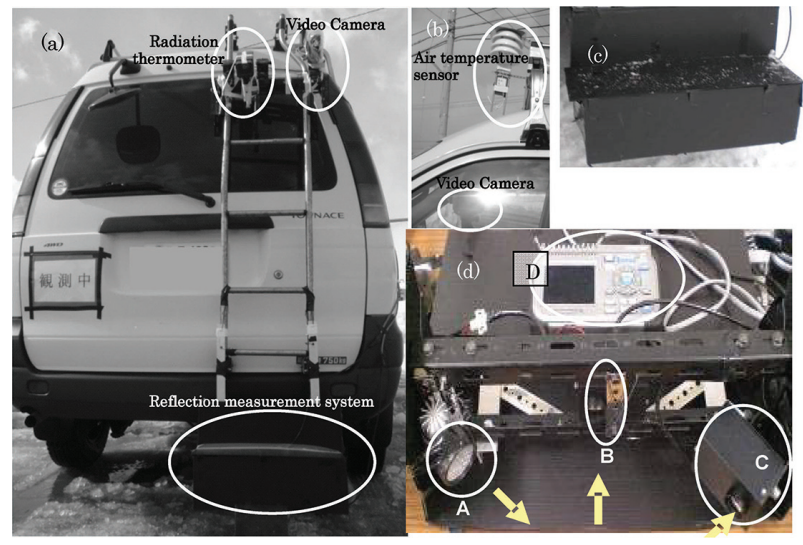

Fig. 8. Mobile reflection measurement system. (a) A reflection measurement system, an infrared radiation thermometer and a video camera were installed at the rear of a car. (b) Air temperature sensor and a front video camera. (c) Appearance of a reflection measurement system. (d) A LED light (A) and two optical sensors (B, C) were set inside of the system. Data was recorded by a data logger (D) in a car.

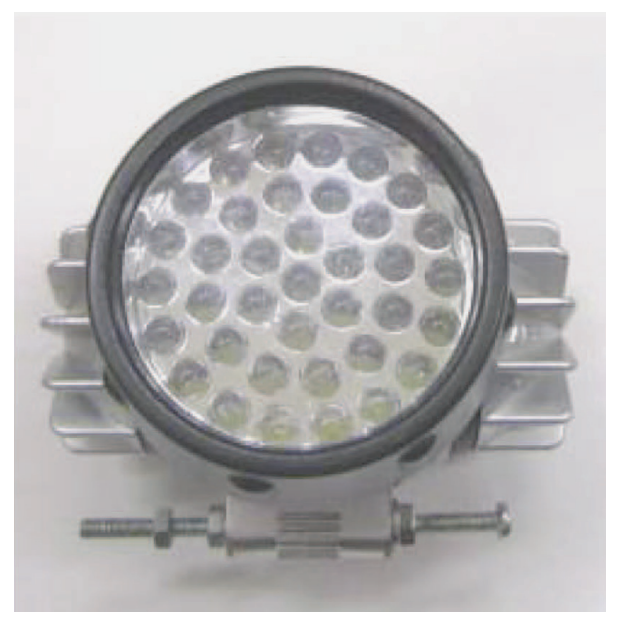

Fig. 9. LED light for a mobile measurement system.

A cap light with 37 LED lights arranged in a 40mm circle was used as the light source (Fig. 9). The directivity of a LED light is not unidirectional, and the light intensity is high $5-10^{\circ}$ from the light axis. This infusibility is not good for exact measurements, but it is good enough for practical measurements of a rough surface when the surface is tilting and the spacing sometimes changes. The light illuminated a measurement surface about $15 \mathrm{~cm}$ in diameter. This light could automatically turn on and off every $0.4 \mathrm{~s}(0.2 \mathrm{~s}$ on and $0.2 \mathrm{~s}$ off), to eliminate outdoor light effects.

\subsection{Elimination of outside light signals}

When we use a reflection measurement system separated from the measured surface, the mixing of outside light becomes a problem and leads to errors in the specular reflection measurements. The effects of the outside light should be eliminated. We attached 
(a)

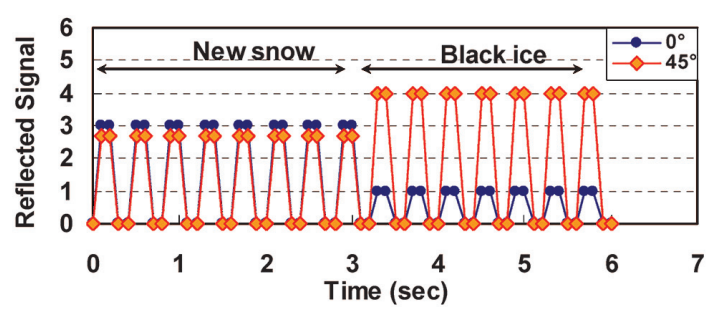

(b)

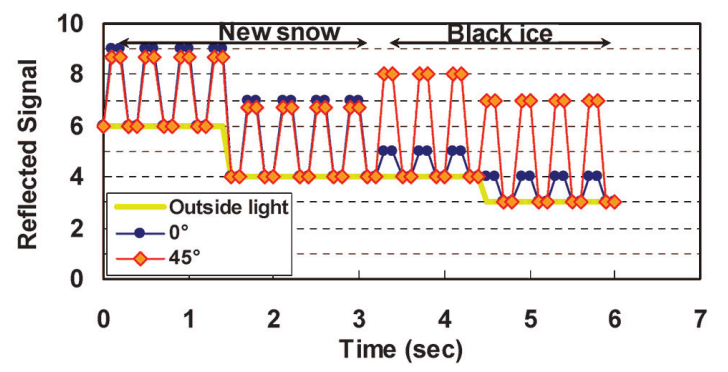

(c)

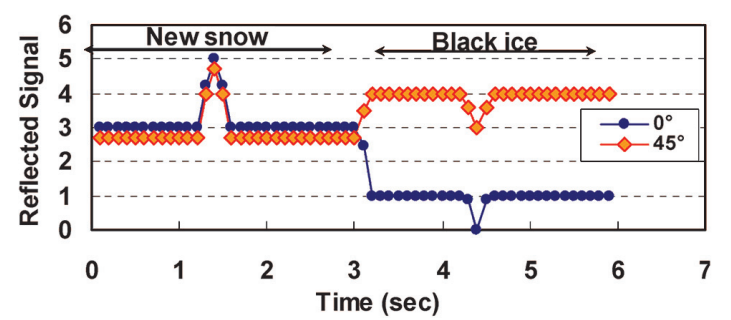

Fig. 10. Virtual surface reflection experiment. (a) Model signals reflected on new snow and black ice surfaces using a blinking light source, with no outside light. (b) Reflection data adding outside light. (c) Standard deviation of four successive data samplings to remove the effects of outside light.

a 10-cm-high soft shade, but this was not sufficient to prevent outside light from entering.

The adopted light could automatically turn on for $0.2 \mathrm{~s}$ and turn off for $0.2 \mathrm{~s}$, and this provided sufficient for eliminating the outside light effects. The method for separating reflection signals and outside light signals was as follows. We set up a virtual surface reflection experiment while driving on a new snow surface and black ice (Fig. 10). The reflection signal at $0^{\circ}$ and $45^{\circ}$ of each $0.1 \mathrm{~s}$ of the experiment when the LED light was blinking at an interval of $0.4 \mathrm{~s}$ is shown in Fig. 10 (a). If some outside light was added to the signal, the signal increased, and the magnitude of the signal due to the outside light could be determined when the LED light was off (Fig. 10 (b)). We determined the amplitudes of the flickering coming from the LED light reflection due to the light turning on and off from the standard deviation $(S D)$ of sequential data. For $n$ data points in an on/off cycle and signals of $\mathrm{S}$ and zero for the light turned on and off, respectively, the $S D$ is

$$
\begin{aligned}
S D & =\sqrt{\frac{1}{\mathrm{n}-1} \sum(x-\bar{x})^{2}}=\sqrt{\frac{\mathrm{n}}{\mathrm{n}-1}\left(\frac{1}{\mathrm{n}} \Sigma(x-\bar{x})^{2}\right)} \\
& =\sqrt{\frac{\mathrm{n}}{\mathrm{n}-1}\left(\frac{S}{2}\right)^{2}}=\sqrt{\frac{\mathrm{n}}{4(\mathrm{n}-1)}} S,
\end{aligned}
$$

from which the signal $S$ can be estimated as follows:

$$
S=\sqrt{\frac{4(n-1)}{n}} S D
$$

In this virtual experiment (period: $0.4 \mathrm{~s}$, logging interval: $0.1 \mathrm{~s}$ ), the number of data points was 4 and $S$ could be estimated from the $S D$ as $S=\sqrt{3} S D$ using Eq. (7).

Figure 10 (c) shows a reproduction of $S$ from the data in Fig. 10 (b) using Eq. (7). The results reproduced the initial reflection data in Fig. 10 (a) well, except when the outside light changed at $1.3 \mathrm{~s}$ and $4.3 \mathrm{~s}$. This virtual experiment therefore demonstrated that the $S D$ reproduced the original reflection signal while the outside light was constant or when it did not change rapidly.

\subsection{Other instrumentation}

As shown in Figure 8, an infrared radiation thermometer was installed at the rear of the vehicle to determine the road surface temperature; it examined the surface about $1 \mathrm{~m}$ behind the optical system. A thermo-recorder was also installed on the car roof to determine the air temperature. Recognition of roadfreezing conditions was also recorded visually; two video cameras were placed at the rear and the front of the car to confirm the results. A data recorder was placed inside the car to collect data from the two photo-diode signals and from the radiation thermometer at 0.2 -s intervals.

\subsection{Mobile observations}

Mobile observations were made along Route 39 around Sekihoku Pass (1050m in elevation) in Hokkaido on March 7, 2009. The observation route is shown in Fig. 11. The numbers from 1) to 9) with elevations are from road-guide signs for drivers.

The lower part of the route was almost straight, but the higher part wound through a mountainous region. Measurements started at 12: 23 at Kita Kitsune Mura (a souvenir shop, $500 \mathrm{~m}$ a.s.1.) and ended at 12: 46 at Sekihoku Pass (1050 m a.s.1.). The times in Fig. 11 indicate the observation times when the car passed that point. The vehicle ran at near $50 \mathrm{~km} \mathrm{~h}^{-1}$. The weather was fine and sunny.

\subsection{Observation results}

The observation results are shown in Fig. 12. These include the road conditions, elevation, road surface temperature, $0^{\circ}$ reflection $\left(S_{0}\right.$ : diffusive reflection), $45^{\circ}$ reflection $\left(S_{45}\right)$, and $45^{\circ}-0^{\circ}$ reflection $\left(S_{45}-S_{0}=R_{\mathrm{S}}\right)$. 


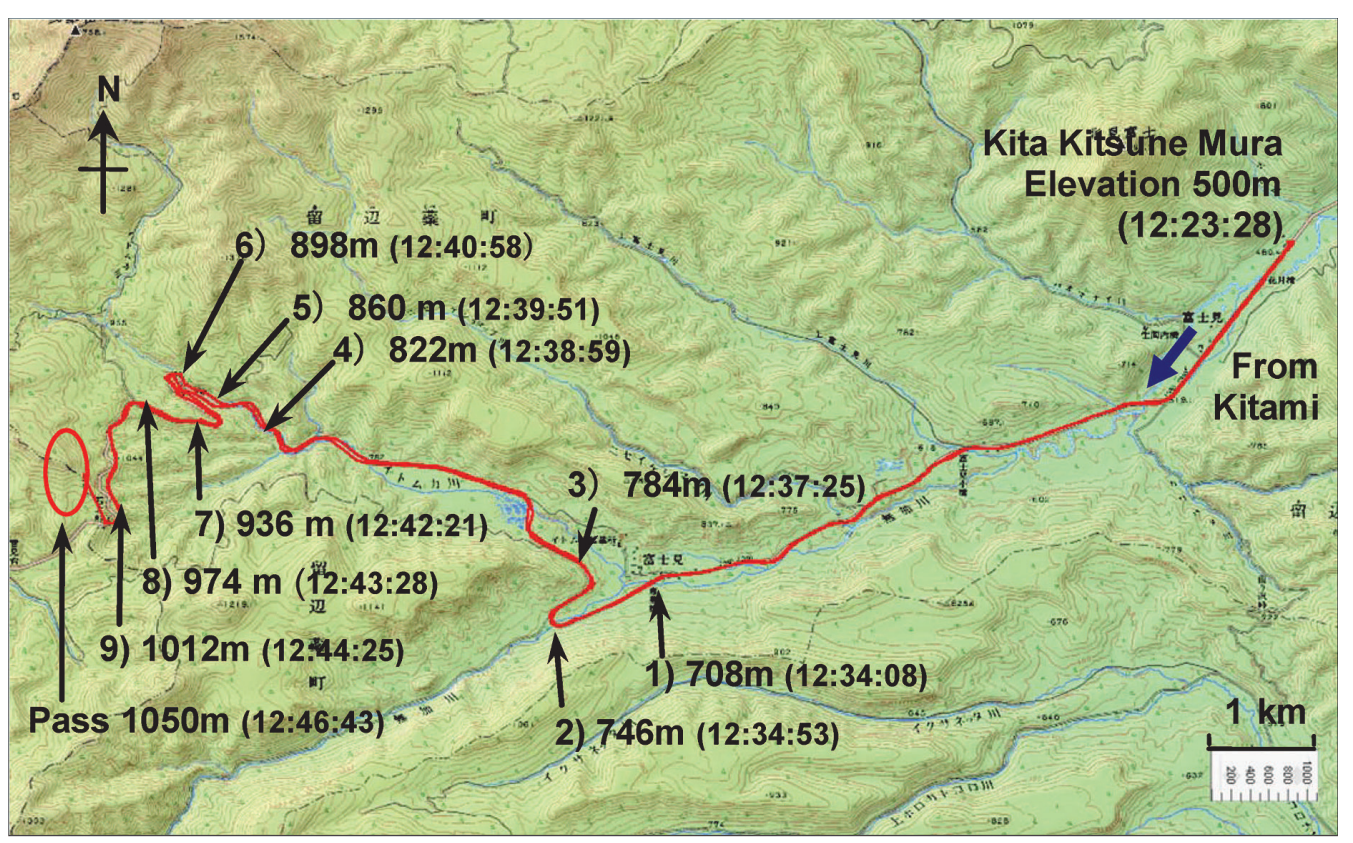

Fig. 11. Observation route in the Sekihoku Pass area, in central Hokkaido. A sensor check was done in the early winter season at several sites around Sekihoku Pass on December 6, 2008, and mobile observations were made along the road on March 7, 2009. The observation time on March 7, 2009 is indicated in the figure.

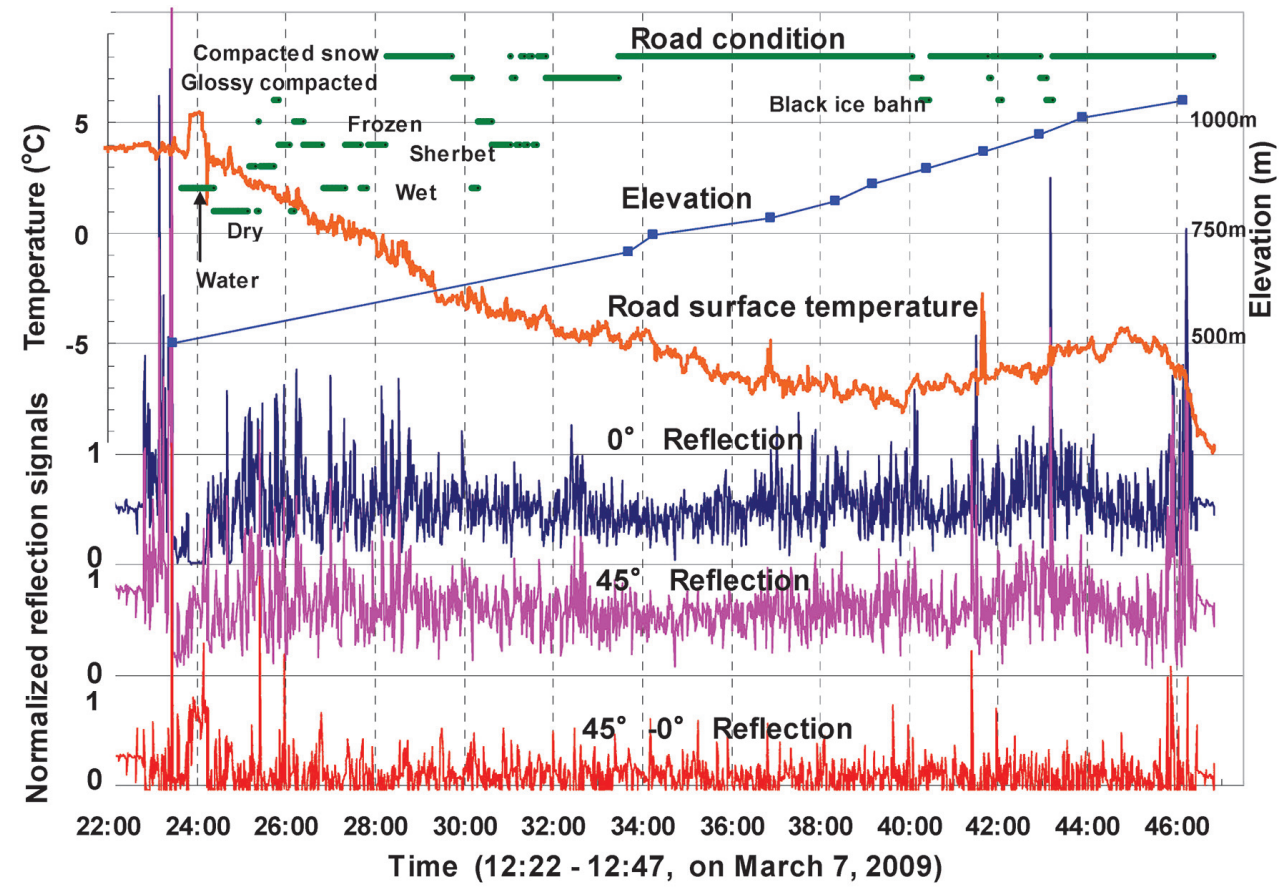

Fig. 12. Mobile observation data along Route 39 around Sekihoku Pass on March 7, 2009. Data are the road conditions, elevation, road surface temperature, $0^{\circ}$ reflection ( $S_{0}$ : diffusive reflection), $45^{\circ}$ reflection $\left(S_{45}\right)$, and $45^{\circ}-0^{\circ}$ reflection $\left(S_{45}-S_{0}=R_{\mathrm{S}}\right)$ from the upper to the lower in a graph.

The road surface temperature was $4^{\circ} \mathrm{C}$ at first $(500 \mathrm{~m}$ a.s.1.), $0^{\circ} \mathrm{C}$ at an elevation of $600 \mathrm{~m}$ a.s.l., and $\left(10^{\circ} \mathrm{C}\right.$ at Sekihoku Pass (1050 $\mathrm{m}$ a.s.l.). The temperature was higher, $5^{\circ} \mathrm{C}$, at around $12: 24$, when the car stopped at a puddle on the road surface for $20 \mathrm{~s}$.

When the surface temperature $T_{\mathrm{S}}$ was positive or $0^{\circ} \mathrm{C}$ (below $600 \mathrm{~m}$ a.s.l), the road conditions were dry, wet, or sherbet; and when $T_{\mathrm{S}}$ was below $0^{\circ} \mathrm{C}$, the condi- tions were mainly compacted snow, sometimes glossy compacted snow, and rarely black ice.

The $0^{\circ}$ reflection $S_{0}\left(=R_{\mathrm{D}}\right.$ : diffusive reflection) and the $45^{\circ}$ reflection $S_{45}$ varied with the road-surface conditions. In the puddle at around $12: 24, S_{45}$ was large while $S_{0}$ was almost zero. The specular reflectance $R_{\mathrm{S}}\left(45^{\circ}-0^{\circ}\right.$ reflection: $\left.S_{45}-S_{0}=R_{\mathrm{S}}\right)$ responded to a glossy surface: see, for example, black ice at 12:40 


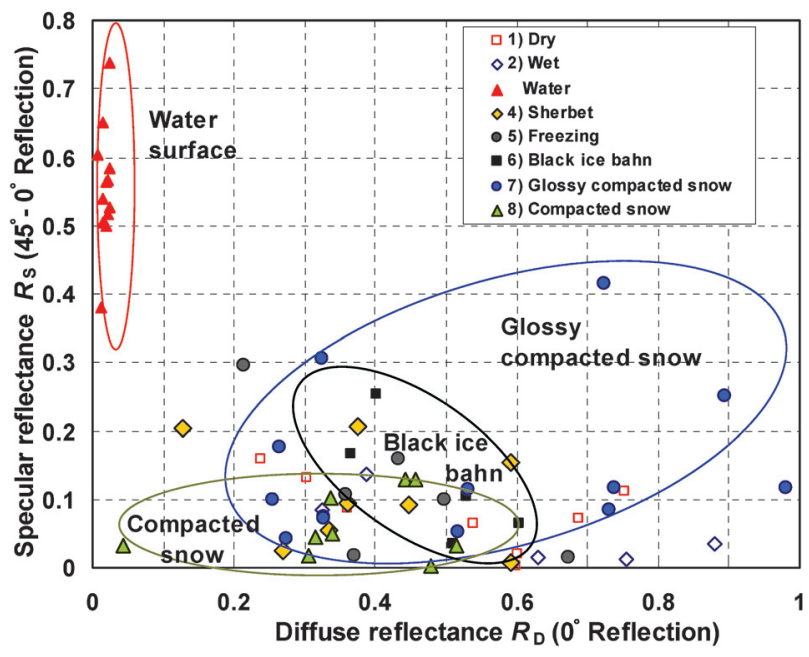

Fig. 13. Specular reflectance $R_{\mathrm{S}}\left(S_{45}-S_{0}\right)$ and diffuse reflectance $R_{\mathrm{D}}\left(=S_{0}: 0^{\circ}\right.$ reflection) of a road surface around Sekihoku Pass on March 7, 2009.

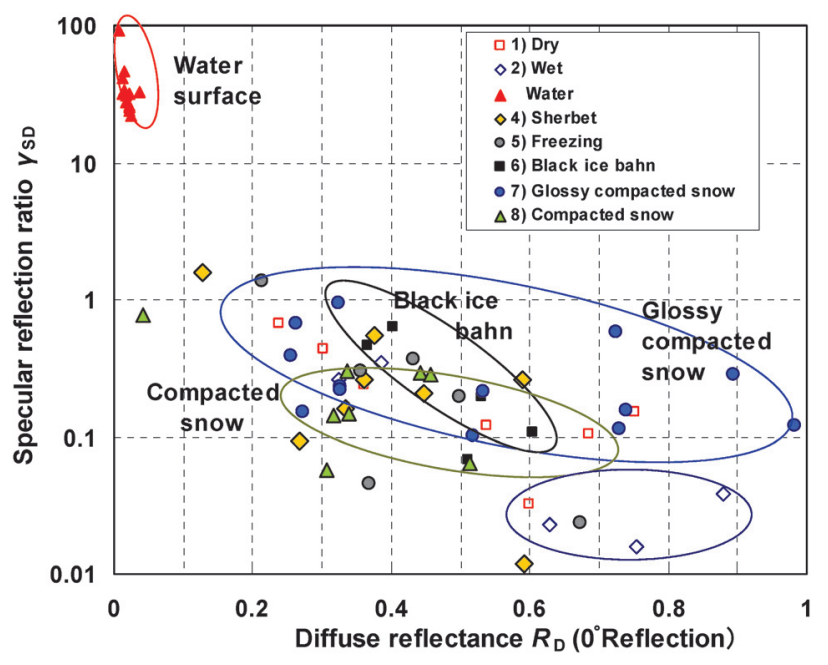

Fig. 14. Specular reflection ratio $\gamma_{\mathrm{SD}}\left(=R_{\mathrm{S}} / R_{\mathrm{D}}\right)$ and diffuse reflectance $R_{\mathrm{D}}$ on March 7, 2009.

and 12:42 and the water surface at 12:24.

\subsection{Specular reflection and road conditions}

The relationship between the specular reflectance $R_{\mathrm{S}}\left(=S_{45}-S_{0}\right)$ and diffuse reflectance $R_{\mathrm{D}}\left(=S_{0}: 0^{\circ}\right.$ reflection) is shown in Fig. 13 from our observations on March 7, 2009 around Sekihoku Pass. The water surface, naturally, showed a large specular reflectance $R_{\mathrm{S}}$ of $0.4-0.7$. The $R_{\mathrm{S}}$ of glossy compacted snow and black ice was $0.03-0.3$, while that of compacted snow and wet surfaces was less than 0.1 .

The relationship between the specular reflection ratio $\gamma_{\mathrm{SD}}\left(=R_{\mathrm{S}} / R_{\mathrm{D}}\right)$ and diffuse reflectance $R_{\mathrm{D}}\left(=S_{0}: 0^{\circ}\right.$ reflection) is shown in Fig. 14, in which the vertical axis is logarithmic; the data are from Fig. 13. The ratio $\gamma_{\mathrm{SD}}$ on the water surface was very large, 30-100, because $R_{\mathrm{D}}$ was very small. The $\gamma_{\mathrm{SD}}$ ranged from $0.1-$ 1.0 on black ice and glossy compacted snow, and was around 0.1 for compacted snow and less than 0.1 for a

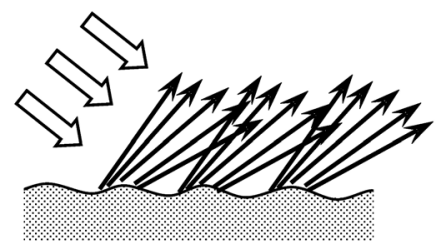

(a)

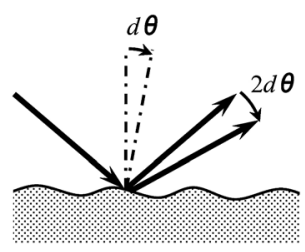

(b)
Fig. 15. Reflection on a rough surface. (a) Dispersed reflection on a wavy surface, (b) Reflection angle change with surface tilting.

wet surface. The ratio for a sherbet surface had a wide range of $0.1-1.0$.

\section{Discussion}

\subsection{Surface smoothness}

The measurements of specular glossiness indicated in JIS Z8741 (1997) are assumed to be of a smooth surface. However, a slippery road surface is glossy, but not completely smooth. It will typically have some roughness, with a wavelength of several $\mathrm{cm}$ to several tens of $\mathrm{cm}$, and reflected light is dispersed (Fig. 15 (a)). When a surface tilts, the normal direction of the surface tilt is $d \theta$, and the reflection angle changes by 2 $d \theta$ (Fig. 15 (b)). If the surface is a sinusoidal wave, with a wavelength of $\lambda$ and relative height of $H$, the reflection angle changes by $\pm 2 \pi \mathrm{H} / \lambda$. From this relationship, a wave with $\mathrm{H} / \lambda$ of $1 / 30$ changes the angle by \pm $12^{\circ}$.

To detect a glossy road surface, we need to measure the dispersed reflected light. One method is to use a sensor with a wide receiving angle; another is to use a light source with a wide area, not a single ray. We used a light source consisting of multiple LED lights for the mobile observations to measure the specular reflectance of the road surface. This technique did not give the exact specular reflectance standardized by JIS Z8741 (1997), but was effective in determining the road surface reflection.

\subsection{Specular reflection}

Comparing Figs. 6 and 13, the specular reflectance $R_{\mathrm{S}}$ was larger than 0.1 on black ice and glossy compacted snow in both cases, so we could distinguish a glossy surface by a threshold $R_{\mathrm{S}}$ of 0.1 . The specular reflection ratio $\gamma_{\mathrm{SD}}$ observed with the portable measurement system (Fig. 7) indicated that the roadsurface conditions were well distinguished by a $\gamma_{\mathrm{SD}}-R_{\mathrm{D}}$ graph, which enhanced indications of the existence of black ice. However, in the same $\gamma_{\mathrm{SD}}-R_{\mathrm{D}}$ graph, shown in Fig. 14, the glossy surfaces and black ice both had large $\gamma_{\mathrm{SD}}$ values, but the black ice was not discriminated from glossy compacted snow. One reason was the accuracy of the $R_{\mathrm{D}}$ measurements because of the standard deviation method used to eliminate the effe- 
cts of outside light; as a result, the estimated value could be overestimated because of noise signals. The $R_{\mathrm{D}}$ seemed too large, $0.3-0.6$, rather than below 0.2 . If $R_{\mathrm{D}}$ was less than $0.2, \gamma_{\mathrm{SD}}$ would be larger and the road conditions would be clearer. The overestimation could be caused by the standard deviation method, which tends to be large when the outside signal or the surface brightness changes rapidly.

\subsection{Glossiness of the road surface}

As described in Section 2.2, the glossiness of a water surface is about $46 \%$. Thus, we can roughly estimate the glossiness of a road surface from $R_{\mathrm{S}}$. In our experiment, the average $R_{\mathrm{S}}$ of water was about 0.55 (Fig. 13), approximately equivalent to a glossiness of $48 \%$. Thus, the glossiness $G$ can be estimated from $R_{\mathrm{S}}$ as $G=87 R_{\mathrm{S}}(\%)$, by which the criterion of a glossy surface as $R_{\mathrm{S}}=0.1$ corresponds to $G=8.7 \%$.

Although, ideally, the glossiness and specular reflectance should be measured by an exact method with a straight light source, no outside light, and a narrow light-receiving angle, as indicated in JIS (1997), the measurement system for our observations provided rough measurements. Although the observed values were not absolute, the system must be available for practical outdoor use on a vehicle.

\subsection{Outside light signal}

A problem with mobile observations is the mixing of outside light into the reflectance measurement system. To eliminate the effects of outside light signals, we adopted a standard deviation method, using Eq. (7) for the flickering light signal, but this introduced an error when the outside light signals changed.

In the mobile observations shown in Fig. 12, the outside light signal was 5 -fold larger than the flickering signal, and the signal-to-noise $(\mathrm{S} / \mathrm{N})$ ratio was 0.2 . Several methods may be used to reduce the error. If we have signals for the light on and off, we can distinguish between the reflected signals when the light is turned on and the outside light signals when light is turned off. Alternatively, if we use an infrared light source and sensors responsive to the same infrared wavelength, we may be able to eliminate the error from outside light signals in the visible wavelength range. As the next step in the development of a real-time alarm system, it would be effective to use an electrical circuit to detect the alternate components of the flickering signals, making real-time measurements possible and providing an alarm system for slippery road surfaces.

\section{Concluding remarks}

1) Observations using a portable measurement system According to our observations made using the portable reflection measurement system, the road conditions were well distinguished by the relationship between the specular reflectance $R_{\mathrm{S}}$ and the diffuse reflectance $R_{\mathrm{D}}$. The measurement system consisted of a light source with an incident angle of $45^{\circ}$ and two photodiode sensors with receiving angles of $0^{\circ}$ for $R_{\mathrm{D}}$ and $45^{\circ}$ for $R_{\mathrm{S}}$. The $R_{\mathrm{S}}$ could be used to judge the degree of gloss of a surface. The $R_{\mathrm{S}}$ values of black ice and glossy compacted ice surfaces were larger than 0.1 , while those of compacted snow and dry surfaces were below 0.05 . The $R_{\mathrm{D}}$ responded to the whiteness of a surface, and the observed $R_{\mathrm{D}}$ showed reasonable values, changing with color.

2) Observations using a mobile (vehicle-based) measurement system

A mobile measurement system was installed at the rear of a car, and continuous observations of $R_{\mathrm{S}}$ and $R_{\mathrm{D}}$ were made in a mountainous region along Route 39 around Sekihoku Pass, Hokkaido. One problem with the mobile observations was the mixing of outside light in the reflectance measurement system. To eliminate outside light signals, we used a flickering light and analyzed the data to determine the specular reflection signal based on a standard deviation method. Using this method, reasonable reflection data were obtained from wet and sherbet surfaces on the lower part of the course and compacted snow and glossy compacted snow around Sekihoku Pass. The specular reflectance $R_{\mathrm{S}}$ was $0.1-0.4$ on glossy compacted snow and black ice, while it was less than 0.1 on compacted snow and wet surfaces. These results were the same as those obtained with the portable measurement system. The diffuse reflectance $R_{\mathrm{D}}$, however, seemed too large, especially for dark surfaces, which was likely due to a tendency of the standard deviation method to show large values when the outside light intensity or the surface brightness changed.

3) Specular reflection and road-surface conditions

The road-surface conditions were discriminated on a graph of specular reflectance $R_{\mathrm{S}}$ and diffuse reflectance $R_{\mathrm{D}}$. Glossy compacted snow and black ice had large $R_{\mathrm{S}}$ values, while the $R_{\mathrm{D}}$ of glossy compacted snow was high and that of black ice was low. The $R_{\mathrm{D}}$ of wet, sherbet, and compacted snow surfaces increased in that order. Although a wet surface appeared near black ice in a $R_{\mathrm{S}}-R_{\mathrm{D}}$ graph, the surface temperature, $T_{\mathrm{S}}$, determined using an infrared radiation thermometer, could readily discriminate a wet surface (positive $T_{\mathrm{S}}$ ) from black ice (negative $T_{\mathrm{S}}$ ).

4) Specular reflection ratio to diffuse reflection

The ratio of specular reflectance to diffuse reflectance, $\gamma_{\mathrm{SD}}\left(=R_{\mathrm{S}} / R_{\mathrm{D}}\right)$, enhanced the indication of black ice on the surface, because $R_{\mathrm{S}}$ was large and $R_{\mathrm{D}}$ was small. This tendency was apparent in the observa- 
tions made with the portable measurement system, although it was not clear in the mobile observations because of the overestimated $R_{\mathrm{D}}$. If we could better eliminate the outside light signals, the ratio of specular reflectance to diffuse reflectance, $\gamma_{\mathrm{SD}}$, may provide a good index of the presence of black ice.

\section{Acknowledgements}

We thank the members of the Civil Engineering Research Institute for Cold Region for valuable discussions. Many thanks are due to Ryota Kobayashi for help with the measurements and data analysis, and to members of the Hokkaido branch of the Japanese Society of Snow and Ice who continued to encourage us. We greatly appreciate the valuable comments from anonymous reviewers and patient help from the editor of our paper.

\section{References}

Alimasi, N. and Takahashi, S. (2009): Kougaku sensor wo mochiita Romentouketsu kenchiki kaihatsu no kenkyu (Development of road freezing detection system by using optical sensor), in Japanese. Hokkaido no Seppyo, 28, pp. 89-92.

Al-Qadi, I. L., Roosevelt, D. S., Decker, R., Wambold, J.C. and Nixon, W. A. (2002): Feasibility of using friction indicators to improve winter maintenance operations and mobility. NCHRP Web Document 53 (Project 6-14): Contractor's Final Report, $151 \mathrm{pp}$.

BMT Fleet Technology Limited (2008): Runway friction characteristics measurement and aircraft braking (RuFAB). Research Project EASA, 148 pp.

Feng, F. and Fu, L. (2008): Evaluation of two new Vaisala sensors for road surface conditions monitoring. Ministry of Transportation. Provincial Highways Management Division Report, Highway Infrastructure Innovation Funding Program, HIIFP-054, 40 pp.

Funahashi, M., Tokunaga, R. A., Takahashi, N. and Asano, M. (2007): Study of friction measuring method on snowy/ icy roads. Presentation at the 8th International Symposium on Cold Region Development (ISCORD 2007).

Haavasoja, T. and Pilli-Sihvola, Y. (2010): Friction as a measure of slippery road surfaces. Proceedings of the 15th SIR WEC Conference, $8 \mathrm{pp}$.

Hamilton, O. (2008): Winter maintenance performance measurement using friction testing. Transportation association of Canada, 211 pp.

Hanatsuka, Y., Motoyama, H. and Yakao, Y. (2009): Discrimination of road-surface condition with sensing technology tire. Presentation at the JSSI \& JSSE Joint Conference, 2009.

Japanese Standards Association (1997): Specular glossiness - Methods of measurement. JIS Z 8741, Tokyo, $18 \mathrm{pp}$.

Matsushima, T., Matsuda, Y. and Kajiya, Y. (2007): Douro riyousya no koutsuu koudou ishiki wo kouryoshita toukidourojyohou no jyujitsu (in Japanese). Kanchi Doboku Kenkyusyo Geppo, 655, pp. 33-39.

Nonstad, B. (2002): The use of friction measurement techniques in winter maintenance in Norway, 11th International Winter Road Congress, CD-ROM, 10 pp.

Takahashi, S. and Alimasi, N. (2009): Shasaigata Romen touketsu kenchi system no shisaku kaihatsu. Proceedings of Cold Region Technology Conference, 25, 09-I-002.

Takahashi, N., Tokunaga, R. A. and Asano, M. (2010): Performance measurement of winter road management. Presentation at the XIIIth International Winter Road Congress.

Tokunaga, R., Funahashi, M., Takahashi, N. and Asano, M., (2008): A feasibility study on friction for winter road management. Transportation Research Board E-C126: Snow 08-020, pp. 352-363.

Wallman, C. G. and Åström, H. (2001): Friction measurement methods and the correlation between road friction and traffic safety: A literature review. Swedish National Road and Transport Research Institute, Project Code 80435, pp. 147. 